\title{
HIGH-RATE DISTRIBUTED MULTI-SOURCE COOPERATION USING COMPLEX FIELD CODING
}

\author{
Alfonso Cano ${ }^{1}$, Jesús Gómez-Vilardebón ${ }^{2}$, Ana Isabel Pérez-Neira ${ }^{3}$, Georgios G. Giannakis ${ }^{1}$ \\ ${ }^{1}$ Dept. of ECE, University of Minnesota, USA \\ ${ }^{2}$ Centre Tecnològic de Telecomunicacions de Catalunya, Spain \\ ${ }^{3}$ Dept. of TSC, Universitat Politècnica de Catalunya, Spain
}

\begin{abstract}
A multisource cooperative protocol is developed capable of achieving diversity order up to the number of cooperating users at a high throughput. In this design each source jointly encodes its own new information symbol with the information symbols received from other sources at past instants. Joint encoding is done using linear complex-field coefficients. Throughput analysis shows gains with respect to existing multi-source protocols and approaches the throughput of non-cooperative schemes. Diversity analysis shows that full spatial diversity is achievable. Simulations confirm the analytically established assessments.
\end{abstract}

Index Terms - Cooperative systems, distributed antennas, multiaccess communication, diversity methods.

\section{INTRODUCTION}

Cooperation in the uplink between sources achieves spatial diversity by forming a virtual antenna array (VAA) through distributed coding and signal processing. The basic practical approach to form such VAAs has been for each source to first "locally share" information with all other sources, and then relay this information, or a jointly-coded version thereof, to the destination $[2,4,7]$. This two-stage transmission scheme is assumed whenever co-located space-time codes or beamforming algorithms are employed in a distributed fashion [1].

Clearly, transmitting in two stages, although practicallyappealing, has a price paid in low throughput per source. This simple observation motivates this work. This paper develops a multisource cooperative protocol that, at any given instant, linearly combines new information to be sent by a source with the information received from other sources at past instants. Linear combination of finite-size constellations is possible using linear constellation precoding (LCP) [8]. If properly designed, the linear complex-field coefficients not only enable the transmission of multiple information symbols at the same time, they also enable spatial diversity. Theoretical analysis corroborated with simulations demonstrate the performance gains of this protocol.

This work was supported through collaborative participation in the Communications and Networks Consortium sponsored by the US Army Research Laboratory under the Collaborative Technology Alliance Program, Cooperative Agreement no. DAAD19-01-2-0011. The US Government is authorized to reproduce and distribute reprints for Government purposes notwithstanding any copyright notation thereon. The work of the second and third author was partially supported by the Catalan Government under grant SGR2005-00996, the Spanish Government under grant TEC 2006-10459/TCM and European project ICT-2007.1.1 NEWCOM++ 216715

\section{PROTOCOL DESCRIPTION}

Consider a set of sources $\left\{S_{n}\right\}_{n=1}^{N}$ willing to communicate with a common access point or destination. Information bits of each source are modulated and carried over constellation symbols. Let $\mathbf{x}_{n}:=\left[x_{n}(0), \ldots, x_{n}(K-1)\right]^{T}$ denote the sequence of $K$ symbols, each drawn from a finite-size constellation set $\mathcal{A}_{s}$ at source $S_{n}, n=1, \ldots, N$. Transmissions are arranged in $K+1$ phases. Assume, for now, that at Phase- $k$, $k=1, \ldots, K-1$, each source, say $S_{n}, n=1, \ldots, N$, has available the set of symbols $\left\{x_{m}(k-1)\right\}_{m=1, m \neq n}^{N}$ from all other sources. Source $S_{n}$ constructs a symbol $s_{n}(k)$, which is a linear combination of its $k$-th constellation symbol $x_{n}(k)$ and $\left\{x_{m}(k-1)\right\}_{m=1, m \neq n}^{N}$. The resulting symbol $s_{n}(k)$ at source $S_{n}$ is given by (see Table 1)

$$
s_{n}(k)=\theta_{n n} x_{n}(k)+\sum_{\substack{m=1 \\ m \neq n}}^{N} \theta_{n m} x_{m}(k-1)
$$

where coefficients $\theta_{n 1}, \ldots, \theta_{n N}$ are designed such that, for any two vectors $\mathbf{x}, \tilde{\mathbf{x}} \in \mathcal{A}_{s}^{N}$

$$
\left|\boldsymbol{\theta}_{n}^{T}(\mathbf{x}-\tilde{\mathbf{x}})\right| \neq 0, \forall n
$$

where $\boldsymbol{\theta}_{n}:=\left[\theta_{n 1}, \ldots, \theta_{n N}\right]^{T}$. This condition guarantees that $s_{n}(k)$ in (1) is unique for every possible different set of symbols $x_{n}(k)$ and $\left\{x_{m}(k-1)\right\}_{m=1, m \neq n}^{N}$. This so-called identifiability criterion will be instrumental in this protocol.

Symbol $s_{n}(k)$ is transmitted by source $S_{n}$ to the destination. Transmissions are carried in separate time slots to avoid interference, distributed synchronization tasks and full-duplex capabilities. The total duration of Phase- $k$ is $N$ channel uses (one per source). Let $y_{n}(k)\left(y_{n m}(k)\right)$ denote the signal received at the destination $\left(S_{m}\right)$ when $S_{n}$ transmits $s_{n}(k)$. Signals $y_{n}(k)$ and $y_{n m}(k)$ are given by

$$
\begin{gathered}
y_{n}(k)=h_{n} \sqrt{\rho_{k}} s_{n}(k)+w_{n}(k) \\
y_{n m}(k)=h_{n m} \sqrt{\rho_{k}} s_{n}(k)+w_{n m}(k)
\end{gathered}
$$

where $h_{n} \sim \mathcal{C N}\left(0, \sigma_{n}^{2} \bar{\gamma}\right)\left(h_{n m} \sim \mathcal{C N}\left(0, \sigma_{n m}^{2} \bar{\gamma}\right)\right)$ is the Rayleigh fading coefficient corresponding to the $S_{n}$-to-destination $\left(S_{n}\right.$-to- $\left.S_{m}\right)$ link; $w_{n}(k)\left(w_{n m}(k)\right)$ is the noise term, normalized to be $\mathcal{C N}(0,1)$; and $\rho_{k}$ weights the transmitted average power. The instantaneous output signal-to-noise ratio (SNR) of each $S_{n}$-to-destination $\left(S_{n}\right.$-to- $\left.S_{m}\right)$ link is defined as $\gamma_{n k}:=\rho_{k}\left|h_{n}\right|^{2}\left(\gamma_{n m k}:=\rho_{k}\left|h_{n m}\right|^{2}\right)$ with expected value $\bar{\gamma}_{n k}=\rho_{k} \sigma_{n}^{2} \bar{\gamma}\left(\bar{\gamma}_{n m k}=\rho_{k} \sigma_{n m}^{2} \bar{\gamma}\right)$. 
Table 1. High-rate distributed coding strategy $(N=3)$.

\begin{tabular}{|c||c|c|c|}
\hline & Phase- $k-1$ & Phase- $k$ & Phase- $k+1$ \\
\hline \hline$S_{1}$ & $\theta_{11} x_{1}(k-1)+\theta_{12} x_{2}(k-2)+\theta_{13} x_{3}(k-2)$ & $\theta_{11} x_{1}(k)+\theta_{12} x_{2}(k-1)+\theta_{13} x_{3}(k-1)$ & $\theta_{11} x_{1}(k+1)+\theta_{12} x_{2}(k)+\theta_{13} x_{3}(k)$ \\
\hline$S_{2}$ & $\theta_{21} x_{1}(k-2)+\theta_{22} x_{2}(k-1)+\theta_{23} x_{3}(k-2)$ & $\theta_{21} x_{1}(k-1)+\theta_{22} x_{2}(k)+\theta_{23} x_{3}(k-1)$ & $\theta_{21} x_{1}(k)+\theta_{22} x_{2}(k+1)+\theta_{23} x_{23}(k)$ \\
\hline$S_{3}$ & $\theta_{31} x_{1}(k-2)+\theta_{32} x_{2}(k-2)+\theta_{33} x_{3}(k-1)$ & $\theta_{31} x_{1}(k-1)+\theta_{32} x_{2}(k-1)+\theta_{33} x_{3}(k)$ & $\theta_{31} x_{1}(k)+\theta_{32} x_{2}(k)+\theta_{33} x_{33}(k+1)$ \\
\hline
\end{tabular}

After Phase- $k$, all sources have received a signal from all other sources. Source $S_{m}$ uses $y_{n m}(k)$ received from $S_{n}$ to obtain a estimate $\hat{x}_{n m}^{M L}(k)$ of $x_{n}(k)$ using the following maximum likelihood (ML) criterion (c.f. (4))

$$
\begin{aligned}
& \hat{x}_{n m}^{M L}(k)= \\
& \arg \min _{x \in \mathcal{A}_{s}}\left\|y_{n m}(k)-h_{n m} \sqrt{\rho_{k}}\left(\theta_{n n} x+\sum_{\substack{p=1 \\
p \neq n}}^{N} \theta_{n p} x_{p}(k-1)\right)\right\|^{2}
\end{aligned}
$$

where $h_{n m}$ is assumed to be known at $S_{m}$. For simplicity of explanation, we will here assume that $\hat{x}_{n m}^{M L}(k)=$ $x_{n}(k), \forall m, n, k$; i.e., sources incur in no detection error at any transmission phase. This assumption will be revisited in the next subsection, were inter-source errors will be incorporated ${ }^{1}$.

After Phase- $k$, all sources know $\left\{x_{n}(k)\right\}_{n=1}^{N}$, which are all the new information symbols sent during Phase- $k$. With $\left\{x_{m}(k)\right\}_{m=1, m \neq n}^{N}$ and $x_{n}(k+1)$ available, $S_{n}$ proceeds to Phase- $k+1$.

The transmission protocol is simply initialized by transmitting $s_{n}(0)=x_{n}(0), \forall n$ during Phase- 0 and finishes at Phase- $K$ by transmitting $s_{n}(K) \forall n$ given by

$$
s_{n}(K)=\sum_{\substack{m=1 \\ m \neq n}}^{N} \theta_{n m} x_{m}(K-1) .
$$

Notice that $s_{n}(K)$ only carries past information symbols. The purpose of sending $s_{n}(K)$ is to guarantee that the lasttransmitted symbols are also "diversified" through the channel in the same way the previous symbols were. Notice that coefficients $\rho_{0}$ and $\rho_{K}$ should be properly adjusted so that symbols $x_{n}(0)$ and $x_{n}(K-1)$, transmitted during both Phase- 0 and Phase- $K$, respectively, suffer same error performance as $x_{n}(1), \ldots, x_{n}(K-1), \forall n$.

This protocol described above requires $(K+1) N$ channel uses to transmit $K$ symbols per source. Defining the throughput $\eta$ as the number of transmitted information symbols per source per channel use (spspcu), the throughput of this strategy is then

$$
\eta=\frac{K}{(K+1) N} \text { spspcu. }
$$

For large $K, \eta$ approaches $1 / N$, which is the throughput of non-cooperative sources transmitting over orthogonal channels. The throughput (7) is considerably higher than that of the MSC strategies in $[1,4]$ where throughputs of $1 / 2 N$ spspcu were at best achieved.

\footnotetext{
${ }^{1}$ Note however that in the uplink sources may be closer to each other than with respect to the destination. Thus, assuming better channel between sources than between sources and destination is a legitimate assumption.
}

\subsection{Inter-source errors}

Assume a rather more realistic scenario where the estimated symbols at $S_{m}$ at any given instant $k,\left\{x_{n m}^{M L}(k)\right\}_{n=1, n \neq m}^{N}$ in (5) differ from $x_{n}(k), \forall k, n \neq m$. The forwarding strategy now has to be modified to account for these errors. For that matter, we will resort to selective-forwarding protocols $[1,2,4]$. From $\left\{x_{n m}^{M L}(k)\right\}_{n=1, n \neq m}^{N}$, define $\mathcal{D}_{m}(k):=\left\{n \mid x_{n m}^{M L}(k)=n, n \neq\right.$ $m\}$ as the set of symbol indexes source $S_{m}$ correctly detected at the $k$-th instant ${ }^{2}$. Note that set $\mathcal{D}_{m}(k)$ does not include index $m$. Sources will broadcast one extra bit to inform other sources and the destination whenever $\hat{x}_{n m}^{M L}(k) \neq x_{n}(k) . S_{m}$ will jointly encode the correctly-received symbols with the own next information symbol $x_{m}(k+1)$. Using $\mathcal{D}_{m}(k), s_{m}(k+1)$ at source $S_{m}$ is given by (c.f. (1))

$$
s_{m}(k+1)=\theta_{m m} x_{m}(k)+\sum_{p \in \mathcal{D}_{m}(k)} \theta_{m p} x_{p}(k)
$$

Note that $s_{m}(k+1)$ will always be non-zero even if $\mathcal{D}_{m}(k)=$ $\emptyset$. Symbol $s_{m}(k+1)$ is transmitted as in (3) and (4).

Since the transmitted symbols have been modified, so has to be the detection rule in (5). Define $\mathcal{C}_{n m}(k):=\mathcal{D}_{n}(k) \cap \mathcal{D}_{m}(k)$ as the set of symbol indexes correctly decoded by both $S_{n}$ and $S_{m}$. Likewise, define $\mathcal{F}_{n m}(k):=\mathcal{D}_{n}(k)-\mathcal{D}_{m}(k)$ as the set of symbols correctly decoded by $S_{n}$ but that $S_{m}$ failed to decode. Source $S_{m}$ now employs the following decoder (c.f. (5))

$$
\begin{aligned}
& \hat{\mathbf{x}}_{n m}^{M L}(k)=\underset{\overline{\mathbf{x}} \in \mathcal{A}_{s}^{\left|\mathcal{F}_{n m}(k-1)\right|+1}}{\arg } \min _{n m} \| y_{n m}(k) \\
& -h_{n m} \sqrt{\rho_{k}}\left(\theta_{n n} \bar{x}_{n}+\sum_{p \in \mathcal{F}_{n m}(k-1)} \theta_{n p} \bar{x}_{p}+\sum_{q \in \mathcal{C}_{n m}(k-1)} \theta_{n q} x_{q}(k-1)\right) \|^{2}
\end{aligned}
$$

where $\bar{x}_{p}:=[\overline{\mathbf{x}}]_{p}$. Notice that the identifiability criterion in (2) guarantees that the detector in (9) has a unique minimum with probability 1 . From $\hat{\mathbf{x}}_{n m}^{M L}(k) S_{m}$ can extract $\hat{x}_{n m}^{M L}(k)=$ $\left[\hat{\mathbf{x}}_{n m}^{M L}(k)\right]_{n}$ and update $\mathcal{D}_{m}(k)$. With $\left\{\hat{x}_{n m}^{M L}(k)\right\}_{n=1, n \neq m}^{N}$ and $\mathcal{D}_{m}(k) S_{m}$ can proceed to Phase- $k+1$.

\subsection{Decoding at the destination}

Assuming knowledge of the $S_{n}-D$ link $\forall n$, the ML detection rule that jointly detects all information symbols sent by all sources, compactly expressed as the set $\boldsymbol{x}=\left\{\left\{x_{n}(k)\right\}_{k=1}^{K}\right\}_{n=1}^{N}$,

\footnotetext{
${ }^{2}$ An error detection code is used here. As in, e.g., [1, 2, 4], this error detection code is assumed perfect and incurs in no bandwidth efficiency loss.
} 
is the following:

$$
\begin{aligned}
& \hat{\boldsymbol{x}}^{M L}=\arg \min _{\boldsymbol{x} \in \mathcal{A}_{s}^{K N}}\left\{\sum_{n=1}^{N}\left\|y_{n}(0)-h_{n} \sqrt{\rho_{0}} x_{n}(0)\right\|^{2}\right. \\
& +\sum_{k=1}^{K-1} \sum_{n=1}^{N}\left\|y_{n}(k)-h_{n} \sqrt{\rho_{k}}\left(\begin{array}{c}
\theta_{n n} x_{n}(k)+\sum_{m \in \mathcal{D}_{n}(k-1)} \theta_{n m} x_{m}(k-1) \\
)
\end{array}\right)\right\|^{2} \\
& \left.+\sum_{n=1}^{N}\left\|y_{n}(K)-h_{n} \sqrt{\rho_{K}} \sum_{m \in \mathcal{D}_{n}(K-1)} \theta_{n m} x_{m}(K-1)\right\|^{2}\right\}
\end{aligned}
$$

where the first and last summands correspond to the initialization and finalization phases Phase- 0 and Phase- $K$, respectively. The search in (10) is performed over the set of constellation codewords $\boldsymbol{x}$ size $\left|\mathcal{A}_{s}\right|^{K N}$. This is a general rule for performance-analysis purposes. Its complexity can be reduced. Notice that symbols overlap across phases introducing memory in the transmitted block. Exploiting the fact that the constellation is finite, the Viterbi algorithm can thus invoked to reduce the search over $\left|\mathcal{A}_{s}\right|^{N}$ possible codewords in $K$ stages [3].

\section{PERFORMANCE ANALYSIS}

We start from the pairwise error probability (PEP), $\operatorname{Pr}(\boldsymbol{x} \rightarrow$ $\tilde{\boldsymbol{x}} \mid \mathbf{h}, \tilde{\mathbf{h}})$ defined as the probability of decoding a codeword $\tilde{\boldsymbol{x}} \in \mathcal{A}_{s}^{K N}$ different from the actual transmitted one $\boldsymbol{x}$ and conditioned on the fading coefficients $\mathbf{h}:=\left[h_{1}, \ldots, h_{N}\right]^{T}$ between sources and the destination and $\tilde{\mathbf{h}}:=\left[h_{11}, \ldots, h_{1 N}, \ldots, h_{N N}\right]^{T}$ between sources. The diversity order $d$ of the system is defined as the slope of the logarithm of the average PEP with respect to the channel coefficients as the logarithm of the SNR goes to infinity; i.e.,

$$
d:=\min _{\boldsymbol{x}, \tilde{\boldsymbol{x}} \neq \boldsymbol{x}}\left\{-\lim _{\bar{\gamma} \rightarrow \infty} \frac{\log \mathbb{E}_{\mathbf{h}, \tilde{\mathbf{h}}}[\operatorname{Pr}(\boldsymbol{x} \rightarrow \tilde{\boldsymbol{x}} \mid \mathbf{h}, \tilde{\mathbf{h}})]}{\log \bar{\gamma}}\right\} .
$$

The diversity definition in (11) is independent of $\sigma_{1}^{2}, \ldots, \sigma_{N}^{2}$ $\left(\sigma_{11}^{2}, \ldots, \sigma_{N N}^{2}\right)$, the path-loss coefficients of every sourcedestination (source-source) link or $\rho_{0}, \ldots, \rho_{K}$, the coefficients that weight the average transmitted power.

\subsection{Error-free inter-source links}

For simplicity in exposition, first consider the case when no inter-source errors occur. Define the error vector $\mathbf{e}:=$ $\left[\mathbf{e}^{T}(0), \ldots, \mathbf{e}^{T}(K-1)\right]^{T}$ with $\mathbf{e}(k):=\mathbf{x}(k)-\tilde{\mathbf{x}}(k)$. Note that e has at least one non-zero entry. Using the Chernoff bound, the PEP (which in the error-free case is independent of $\tilde{\mathbf{h}}$ ) can be bounded as (see also $[1,8]$ )

$$
\operatorname{Pr}(\boldsymbol{x} \rightarrow \tilde{\boldsymbol{x}} \mid \mathbf{h}) \leq \exp \left(\alpha\left\|\boldsymbol{\Gamma}_{\mathbf{e}} \mathbf{h}\right\|^{2}\right)
$$

for some finite constant $\alpha$ and with $\Gamma_{\mathbf{e}}$ being the pairwise error matrix defined as:

$$
\boldsymbol{\Gamma}_{\mathbf{e}}:=\left[\begin{array}{c}
\operatorname{diag}(\mathbf{e}(0)) \\
\operatorname{diag}\left(\mathbf{D}_{\theta} \mathbf{e}(1)+\tilde{\mathbf{\Theta}} \mathbf{e}(0)\right) \\
\operatorname{diag}\left(\mathbf{D}_{\theta} \mathbf{e}(2)+\tilde{\boldsymbol{\Theta}} \mathbf{e}(1)\right) \\
\vdots \\
\operatorname{diag}\left(\mathbf{D}_{\theta} \mathbf{e}(K-1)+\tilde{\mathbf{\Theta}} \mathbf{e}(K-2)\right) \\
\operatorname{diag}(\tilde{\boldsymbol{\Theta}} \mathbf{e}(K-1))
\end{array}\right]
$$

where the $N \times N$ matrices $\mathbf{D}_{\theta}$ and $\tilde{\boldsymbol{\Theta}}$ are composed of the diagonal and off-diagonal entries of $\boldsymbol{\Theta}$, respectively, and $[\boldsymbol{\Theta}]_{n m}:=$ $\theta_{n m}$.

The distribution of $\mathbf{h}$ is $\mathbf{h} \sim \mathcal{C N}\left(0, \mathbf{D}_{\sigma_{n}^{2}} \bar{\gamma}\right)$, where $\mathbf{D}_{\sigma_{n}^{2}}:=$ $\operatorname{diag}\left(\left[\sigma_{1}^{2}, \ldots, \sigma_{N}^{2}\right]\right)$. Computing the expected value of (12) wrt h we have that

$$
\mathbb{E}_{\mathbf{h}}[\operatorname{Pr}(\boldsymbol{x} \rightarrow \tilde{\boldsymbol{x}} \mid \mathbf{h})] \leq(\beta \bar{\gamma})^{\operatorname{rank}\left(\boldsymbol{\Gamma}_{\mathbf{e}}\right)}
$$

for some finite constant $\beta$. Plugging (14) in (11), the diversity order $d$ is related to the rank of $\boldsymbol{\Gamma}_{\mathbf{e}}$ as stated in the following proposition $^{3}$.

Proposition 1 Consider the LCP coefficients $\theta_{n m} \forall n, m=$ $1, \ldots, N$ designed to hold (2). The diversity order as defined in (11) of the protocol defined in Section 2 is

$$
d:=\min _{\boldsymbol{x}, \tilde{\boldsymbol{x}} \neq \boldsymbol{x}}\left\{\operatorname{rank}\left(\boldsymbol{\Gamma}_{\mathbf{e}}\right)\right\}=N
$$

Thus, in the error-free case, this protocol achieves the maximum diversity order, equal to the number of sources $N$.

\subsection{Errors in inter-source links}

The diversity order in this case is found in two steps. Firstly, the probability of having a given set of error events at the sources is found. Secondly, the PEP at the destination given that this set of errors happened (which we name the error-conditional PEP), is found. Define $\mathcal{E}_{n}(k)$ as the set of sources that failed to detect $x_{n}(k)$; i.e., $\mathcal{E}_{n}(k):=\left\{m \mid n \notin \mathcal{D}_{m}(k), m \neq n\right\}$. Notice that by construction $n \notin \mathcal{E}_{n}(k)$. The set of all decoding errors at all transmissions times are packed in the super-set $\mathcal{E}:=\left\{\left\{\mathcal{E}_{n}(k)\right\}_{n=1}^{N}\right\}_{k=0}^{K-1}$. Next, define $\operatorname{Pr}(\boldsymbol{x} \rightarrow \tilde{\boldsymbol{x}} \mid \mathbf{h}, \mathcal{E})$ as the $\mathrm{PEP}$ at the destination conditioned on a set $\mathcal{E}$ of decoding errors at the sources. Likewise, define $\operatorname{Pr}(\mathcal{E} \mid \tilde{\mathbf{h}})$ as the probability of having a set $\mathcal{E}$ of errors, conditioned on the inter-source channel coefficients $\tilde{\mathbf{h}}$. The PEP can be expressed as a marginalization over all possible error events; i.e. $\operatorname{Pr}(\boldsymbol{x} \rightarrow \tilde{\boldsymbol{x}} \mid \mathbf{h}, \tilde{\mathbf{h}})=$ $\sum_{\forall \mathcal{E}} \operatorname{Pr}(\boldsymbol{x} \rightarrow \tilde{\boldsymbol{x}} \mid \mathbf{h}, \mathcal{E}) \operatorname{Pr}(\mathcal{E} \mid \tilde{\mathbf{h}})$ with expected value

$$
\begin{aligned}
& \mathbb{E}_{\mathbf{h}, \tilde{\mathbf{h}}}[\operatorname{Pr}(\boldsymbol{x} \rightarrow \tilde{\boldsymbol{x}} \mid \mathbf{h}, \tilde{\mathbf{h}})]= \\
& \sum_{\forall \mathcal{E}} \mathbb{E}_{\mathbf{h}}[\operatorname{Pr}(\boldsymbol{x} \rightarrow \tilde{\boldsymbol{x}} \mid \mathbf{h}, \mathcal{E})] \mathbb{E}_{\tilde{\mathbf{h}}}[\operatorname{Pr}(\mathcal{E} \mid \tilde{\mathbf{h}})]
\end{aligned}
$$

Both factors in the right-hand side of (16) can be found independently for a given (fixed) error event $\mathcal{E}$. First we start with $\mathbb{E}_{\mathbf{h}}[\operatorname{Pr}(\mathcal{E} \mid \tilde{\mathbf{h}})]$. From the detector (9), and after applying the Chernoff bound, the conditional probability that source $S_{m}$ fails to detect $x_{n}(k)$ can be bound as $\operatorname{Pr}(m \in$ $\left.\mathcal{E}_{n}(k) \mid \tilde{\mathbf{h}}\right) \leq \exp \left(\alpha_{n m}(k)\left|h_{n m}\right|^{2}\right)$ for some finite coefficient $\alpha_{n m}(k)$. Due to conditional independence, the conditional probability of having $\mathcal{E}$ errors is thus $\operatorname{Pr}(\mathcal{E} \mid \tilde{\mathbf{h}}) \leq$ $\exp \left(\sum_{k=0}^{K-1} \sum_{n=1}^{N} \sum_{m \in \mathcal{E}_{n}(k)} \alpha_{n m}(k)\left|h_{n m}\right|^{2}\right)$ with expected value

$$
\begin{aligned}
\mathbb{E}_{\tilde{\mathbf{h}}}[\operatorname{Pr}(\mathcal{E} \mid \tilde{\mathbf{h}})] & \leq\left(\beta^{\prime} \bar{\gamma}\right)^{\sum_{n=1}^{N}\left|\cup_{k=0}^{K-1} \mathcal{E}_{n}(k)\right|} \\
& \leq\left(\beta^{\prime} \bar{\gamma}\right)^{\max _{n}\left\{\left|\cup_{k=0}^{K-1} \mathcal{E}_{n}(k)\right|\right\}}
\end{aligned}
$$

\footnotetext{
${ }^{3}$ Proofs for all the propositions in this paper are omitted due to space limitations.
} 


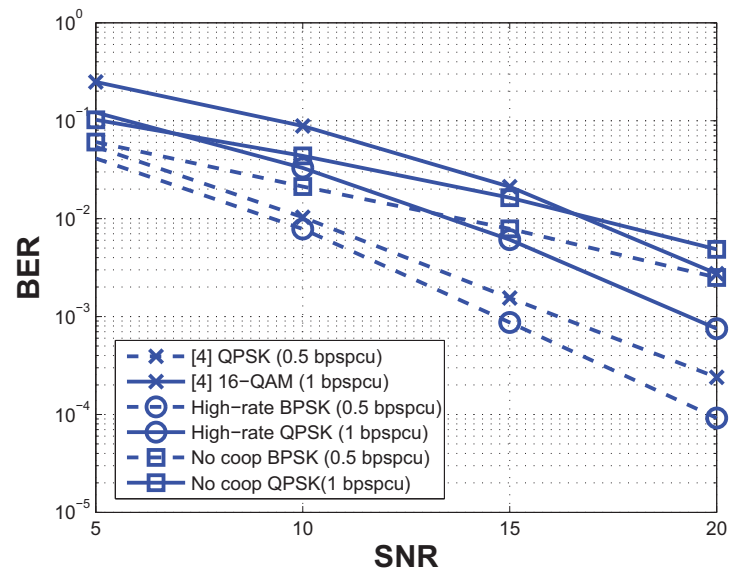

Fig. 1. BER for $N=2$ of the proposed protocol vs. [4] vs. non-cooperative case at different throughputs.

where the second inequality holds at high SNR and for some finite constant $\beta^{\prime}$.

We turn now to the error-conditional PEP at the destination $\operatorname{Pr}(\boldsymbol{x} \rightarrow \tilde{\boldsymbol{x}} \mid \mathbf{h}, \mathcal{E})$. Using again the Chernoff bound, $\operatorname{Pr}(\boldsymbol{x} \rightarrow$ $\tilde{\boldsymbol{x}} \mid \mathbf{h}, \mathcal{E}) \leq \exp \left(\alpha^{\prime}\left\|\boldsymbol{\Gamma}_{\mathbf{e}}(\mathcal{E}) \mathbf{h}\right\|^{2}\right)$ where $\boldsymbol{\Gamma}_{\mathbf{e}}(\mathcal{E})$ is constructed as in (13) setting $\theta_{n m} e_{m}(k)=0$ whenever $m \in \mathcal{E}_{n}(k)$. As in (14), the expected value of the conditional PEP is given by $\mathbb{E}_{\tilde{\mathbf{h}}}[\operatorname{Pr}(\boldsymbol{x} \rightarrow \tilde{\boldsymbol{x}} \mid \mathbf{h}, \mathcal{E})] \leq\left(\beta^{\prime \prime} \bar{\gamma}\right)^{-\operatorname{rank}\left(\boldsymbol{\Gamma}_{\mathbf{e}}(\mathcal{E})\right)}$, for some finite constant $\beta^{\prime \prime}$. Combining this result with (17), a bound on (16) can be found to establish the diversity order of this scheme, as stated in the following proposition.

Proposition 2 Consider the coefficients $\theta_{n m} \forall n, m=1, \ldots, N$ designed to hold (2). The diversity order as defined in (11) of the protocol defined in Section 2.1 is

$$
d:=\min _{\boldsymbol{x}, \tilde{\boldsymbol{x}} \neq \boldsymbol{x}, \mathcal{E}}\left\{\operatorname{rank}\left(\boldsymbol{\Gamma}_{\mathbf{e}}(\mathcal{E})\right)+\max _{n}\left\{\left|\cup_{k=0}^{K-1} \mathcal{E}_{n}(k)\right|\right\}, N\right\}=N
$$

Thus, the diversity order is independent of the sources' error events $\mathcal{E}$ and is equal to the number of sources $N$.

\section{SIMULATIONS}

In this section we present simulations that test the error performance of the proposed cooperative protocol. Matrix $\Theta$ is taken from [8]. Sources-source links are assumed to have the same average $\operatorname{SNR}\left(\tilde{\gamma}_{k}:=\gamma_{11 k}=\ldots=\gamma_{N N k}\right)$, and so are the source-destination links $\left(\gamma_{k}:=\gamma_{1 k}=\ldots=\gamma_{N k}\right)$. However, the SNR in the source-destination link is $3 \mathrm{~dB}$ greater than source-source $\operatorname{SNR}\left(\gamma_{k}=\tilde{\gamma}_{k}+3 \mathrm{~dB}\right)$. The block length is $K=100$ and the Viterbi algorithm is employed for decoding.

Fig. 1 shows the average bit error rate (BER) as a function of the average SNR for $N=2$ sources. Two cases with rates of 0.5 and 1 bits per source per channel use (bpspcu) are considered, employing BPSK and QPSK modulations, respectively. For reference, the BER when sources are not cooperating is also depicted. Also, the BER when sources are implementing the distributed protocol in [4] is included. In this case, for

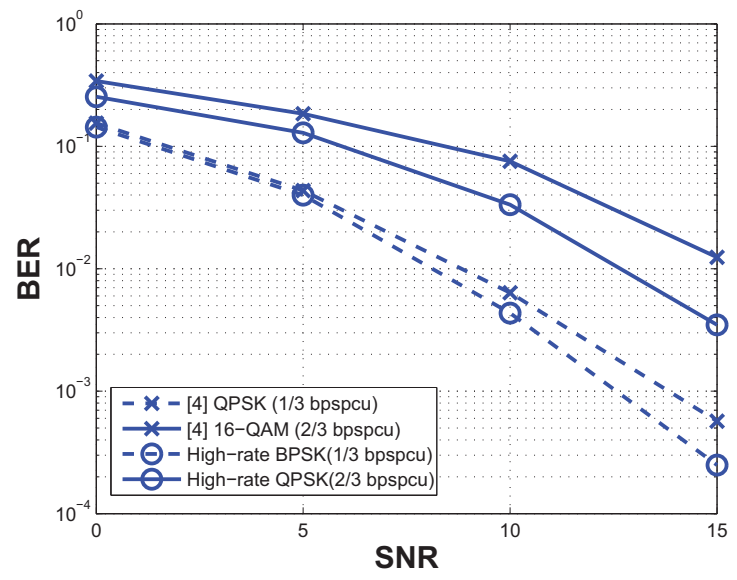

Fig. 2. BER for $N=3$ of the proposed protocol vs. [4] at different throughputs.

a fair comparison, we plot [4] when using QPSK and 16-QAM modulations to achieve the same throughput of 0.5 and $1 \mathrm{bp}-$ spcu, respectively. All protocols transmit with the same average transmit power. We can verify that the slope of the BER is fixed to 2 for both protocols with a higher coding gain of the one developed here due to the use of lower-order constellations. The new design clearly outperforms the non-cooperative case for the same system resources. This same behavior is observed in Fig. 2 when $N=3$ sources are considered with throughputs of $1 / 3$ and $2 / 3$ bpspcu.

\section{REFERENCES}

[1] A. Cano, T. Wang, A. Ribeiro, and G. B. Giannakis, LinkAdaptive Distributed Coding for Multi-Source Cooperation, EURASIP J. on Adv. in Signal Process., submitted April 2007.

[2] J. N. Laneman and G. W. Wornell, "Distributed space-timecoded protocols for exploiting cooperative diversity in wireless networks," IEEE Trans. on Info. Theory, vol. 49, no. 10, pp. 2415-2425, October 2003.

[3] J. G. Proakis, Digital Communications, McGraw-Hill Higher Education, 2001, fourth ed.

[4] A. Ribeiro, R. Wang, and G. B. Giannakis, "Multi-source cooperation with full diversity spectral-efficiency and controllablecomplexity," IEEE J. on Sel. Areas in Commun., vol. 25, no. 2, pp. 415-425, Feb. 2007.

[5] G. Scutari, S. Barbarossa, "Distributed space-time coding for regenerative relay networks" IEEE Trans. on Wireless Commun., vol. 4, no. 5, pp. 2387 - 2399, Sept. 2005.

[6] A. Sendonaris, E. Erkip, and B. Aazhang, "User cooperation diversity - part I: system description," IEEE Trans. on Commun., vol. 51, no. 11, pp. 1927-1938, Nov. 2003.

[7] O. Shalvi, "Multiple source cooperation diversity," IEEE Commun. Letters, vol. 8, pp. 712-714, Dec. 2004.

[8] Y. Xin, Z. Wang, and G. B. Giannakis, "Space-time diversity systems based on linear constellation precoding," IEEE Trans. on Wireless Commun., vol. 2, pp. 294-309, Mar. 2003. 